\title{
On the Effectiveness of Probabilistic Packet Marking for IP Traceback under Denial of Service Attack
}

\author{
Kihong Park Heejo Lee \\ Network Systems Lab \\ Department of Computer Sciences \\ Purdue University \\ West Lafayette, IN 47907 \\ \{park,hlee\}@cs.purdue.edu
}

\begin{abstract}
Effective mitigation of denial of service (DoS) attack is a pressing problem on the Internet. In many instances, DoS attacks can be prevented if the spoofed source IP address is traced back to its origin which allows assigning penalties to the offending party or isolating the compromised hosts and domains from the rest of the network. Recently IP traceback mechanisms based on probabilistic packet marking (PPM) have been proposed for achieving traceback of DoS attacks. In this paper, we show that probabilistic packet marking —of interest due to its efficiency and implementability vis-à-vis deterministic packet marking and logging or messaging based schemes—-suffers under spoofing of the marking field in the IP header by the attacker which can impede traceback by the victim. We show that there is a trade-off between the ability of the victim to localize the attacker and the severity of the DoS attack, which is represented as a function of the marking probability, path length, and traffic volume. The optimal decision problem - the victim can choose the marking probability whereas the attacker can choose the spoofed marking value, source address, and attack volume-can be expressed as a constrained minimax optimization problem, where the victim chooses the marking probability such that the number of forgeable attack paths is minimized. We show that the attacker's ability to hide his location is curtailed by increasing the marking probability, however, the latter is upper-bounded due to sampling constraints. In typical IP internets, the attacker's address can be localized to within 2-5 equally likely sites which renders PPM effective against single source attacks. Under distributed DoS attacks, the uncertainty achievable by the attacker can be amplified, which diminishes the effectiveness of PPM.
\end{abstract}

Keywords - Probabilistic packet marking, Denial of service attack, Traceback analysis, Network security, IP spoofing

\section{INTRODUCTION}

\section{A. Background}

Denial of service (DoS) is a pressing problem on the Internet as evidenced by recent attacks on commercial servers and ISPs and their consequent disruption of services [2]. DoS attacks [3], [4], [5], [6], [7], [8] consume resources associated with various network elements-e.g., Web servers, routers, firewalls, and end hosts-which impedes the efficient functioning and provisioning of services in accordance with their intended purpose. Their impact is more pronounced than network congestion due to the concentrated and targeted nature of resource depletion and clogging, which not only impacts quality of service (QoS) but can affect the very availability of services. Susceptibility to DoS is an intrinsic problem of any service provisioning system - albeit amplified in the networked digital envi-

This work was supported in part by NSF grant EIA-9972883. This is an extended abstract. The full paper is available as a tech-report [1].

K. Park was additionally supported by NSF grants ANI-9714707, ANI9875789 (CAREER), ESS-9806741, ANI-0082861 (ITR), and grants from the Purdue Research Foundation, Santa Fe Institute, Sprint, and Xerox.

H. Lee was additionally supported by the Center for Education and Research in Information Assurance and Security (CERIAS). ronment due to speed and automation-where, at a minimum, the occurrence of a potentially valid event (e.g., service request, TCP SYN packet) must be processed to ascertain its validity. Even though the resource expenditure associated with processing a single event may be negligible, when this is multiplied by the large factors enabled by the high bandwidth of modern broadband networks, its impact can be significant no matter how small the individual processing overhead. Firewalls and filters running at gateway routers can shield a network system from outside DoS flows, but if their function includes selectively admitting valid client flows resident outside a guarded domain, then this very filtering service can be impeded by DoS attacks targeted at the gateway. As with prank telephone calls or ringing of door bells in days gone by, an effective means of preventing DoS attacks from occurring in the first place-also the only fundamental solution given the intrinsic susceptibility of service provisioning systems to DoS-lies in identification of the attacker which admits assigning commensurate costs (e.g., legal or economical) to the perpetrating entity. Even if the attack was instituted from compromised hosts intruded by an attacker, if the physical source of DoS traffic can be identified, then at the very least the invaded network element can be isolated or shut down, and in some instances, the attacker's identity can be further traced back by state information available on the compromised system. In this paper, we address the source identification problem and analyze its properties from a probabilistic packet marking approach, motivated by its appealing feature with respect to efficiency and implementability.

\section{B. A Case for Probabilistic Packet Marking}

A "simple" way of identifying the physical source of DoS traffic is by elimination of IP address spoofing. If all ISPs were to implement mechanisms for preventing IP source address spoofing - which is, technically, easy to do-then source identification (also called IP traceback in [9]), would be solved. A less drastic measure, based on packet marking, would allow spoofed packets to pass through, however, with the corrected source IP address overwriting the spoofed source IP address. For various practical reasons, this may be difficult to achieve or require a prolonged period to be broadly deployed on the Internet. Thus, there is a need for incrementally deployable techniques that may not completely eliminate the DoS problem, but reduce it to a "manageable" level.

A number of recent works have studied the problem of trac- 
ing the physical source of a DoS attack [6], [9], [10], [11], [12], [13], [14]. In deterministic packet marking [15], the source of a traffic flow is recovered by employing tracing information inscribed in the packet. Packet marking can be viewed as a form of "stateless logging" which emulates the capability of path recovery by router based information logging [12], [14], without incurring the latter's statefulness and associated space overhead. A related method is messaging based path recovery [10] which uses control messages emitted from routers conveying path information to destination nodes. Thus (router) statelessness is achieved, however, at the cost of message overhead. Packet marking — and, to some extent, messaging — follows the end-toend paradigm [16] where complexity of path recovery is pushed to the edge while imposing a minimal footprint on per-hop network support requirements.

A significant drawback of deterministic packet marking (DPM) is the increasing packet header size requirement which grows linearly with hop count. In addition to amplifying packet size - a form of communication complexity-dynamically variable packet sizes complicate router processing which can impart nontrivial overhead to achieving terabit-per-second switching speeds. In probabilistic packet marking [9], each router probabilistically inscribes its local path information onto a traversing packet so that the destination node (i.e., victim of an attack) can reconstruct, with high probability, the complete path traversed by inspecting the markings on the received packets, assuming the attack volume is sufficiently high. This corresponds to probabilistically "sampling" the route undertaken by an attack using constant space in the packet header independent of hop count, which provides the key advantage over deterministic packet marking. In probabilistic marking, when a router decides to mark based on a coin toss with marking probability $p$, it overwrites the information contained in the marking field, thus erasing any possible markings by upstream routers. Thus, for PPM to work, it is necessary that $p<1$. By the same token, with some positive probability, a packet will arrive at the destination without having been marked by any of the intermediate routers. This reveals-above and beyond the need for requiring a set of packets to recover the attack path-a potentially serious weakness of PPM since the marking field may contain a value, inscribed by the attacker, whose aim is to confuse or impede the victim's ability to traceback. In this paper, we give a comprehensive treatment of the spoofed marking field problem.

\section{New Contributions}

We analyze the effectiveness of probabilistic packet marking for IP traceback under DoS attack. Our technical contributions are two-fold.

First, we define the source identification problem in the framework of probabilistic packet marking (PPM) and present a comprehensive analysis of its properties. We show that PPM is vulnerable to spoofing of the marking field in the IP header by the attacker which can impede traceback by the victim. We show that there is a trade-off relation between the ability of the victim to localize the attacker and the severity of the DoS attack, which is a function of the marking probability, path length, and traffic volume. The optimal decision problem - the victim can choose the marking probability and the attacker chooses the spoofed marking value, source address, and attack volumecan be expressed as a constrained minimax optimization problem: the victim selects the marking probability such that the number of forgeable attack paths is minimized and the attacker chooses the traffic volume and marking value to maximize uncertainty. We show that the attacker's ability to hide his location is curtailed by increasing the marking probability, however, the degree to which the victim can delimit the attacker's injection of uncertainty is bounded by sampling constraints. In particular, the attacker, by choosing a minimal attack traffic volume, can amplify the number of equally likely forged attack paths to $d-1$, independent of the victim's choice of marking probability, where $d$ is the path length. In IP internetworks with hop count 25 or less (as is the case on the Internet) and attack volume in the thousands of packets - to qualify as a DoS attack, the victim's resources must be nontrivially taxed-we show that the attacker's address can be localized to within $2-5$ equally likely sites which renders PPM effective against single source attacks.

Second, we analyze the consequences of the attacker mounting distributed DoS attacks where each partaking attack host transmits a minimal traffic volume to maximize anonymity, and attack volume amplification is achieved by engaging a large number of sources. We show that for a given attack volume, by mounting a distributed denial of service attack, the uncertainty injected into IP traceback can be amplified above and beyond the effect afforded by distributedness. Thus PPM, while effective against single-source attacks, is potentially vulnerable when subject to distributed DoS attacks.

The rest of the paper is organized as follows. In the next section, we give a summary of related works. In Section III, we discuss the core issues surrounding source identification and define the IP traceback problem in the framework of PPM. In Section IV, we present the analysis of single-source DoS attack which is complemented by numerical evaluations using Internet related parameters. In Section V we study the distributed DoS case and show its detrimental consequences on PPM. We conclude with a discussion of our results.

\section{RELATED WORK}

Several types of DoS attacks have been identified [2], [4], [6], [7] with the most basic DoS attack demanding more resources than the target system or network can supply. Resources may be network bandwidth, file system space, processes, or network connections [6]. While host-based DoS attacks are more easily traced and managed, network-based DoS attacks which exploit weaknesses of the TCP/IP protocol suite [17], represent a more subtle and challenging threat [6], [9]. Network-based DoS attacks, by default, employ spoofing to forge the source address of DoS packets to hide the identity of the physical source [8]. Previous works have focused on detecting DoS attacks and mitigating their detrimental impact upon the victim [18], [19], [20], [21]. This approach does not eliminate the problem, nor does it deter potential attackers. As a means of preventing networkbased DoS attacks, edge filtering in border gateways has been proposed for limiting IP source address spoofing [22], [23], [24]. The filtering rules can affect dropping of forged packets using egress filtering in user organizations and ingress filtering 
in ISPs [2], [25].

A number of recent works have studied source identification (also called IP traceback in [9]) which span a range of techniques with their individual pros and cons. In link testing, the physical source of an attack is identified by tracing it back hopby-hop through the network [11]. Traceback is typically performed manually and recursively repeated at the upstream router until the originating host is reached. The drawbacks of link testing include multiple branch points, slow traceback during an attack, communication overhead due to message exchange, and administrative constraints between network operators including legal issues [11]. The audit trail approach facilitates tracing via traffic logs at routers and gateways [12], [14], [26]. This method is conducive to off-line traceback of DoS attacks. A principal weakness, however, is the high storage and processing overhead incurred at routers - which are expected to switch at Tbps rates-which can exert a significant burden. In behavioral monitoring, the likely behavior of an attacker during a DoS attack is monitored to identify the source [6]. For example, an attacker may perform DNS requests to resolve the name of the target host which may not be resident in its local name server's cache. During a DoS attack, an attacker may try to gauge the impact of the attack using various service requests including Web and ICMP echo requests. Thus, logging of such events and activities can reveal information about the attacker's source. In packet-based traceback, packets are marked with the addresses of intermediate routers, in some sense, an inverse operation of source routing and similar to the IP Record Route option [27]. The victim uses information inscribed in packets to trace the attack back to its source. A related method is generating information packetsseparate from data packets- that convey analogous path information as ICMP traceback messages to the victim [10]. In both methods, overhead in the form of variable-length marking fields that depend on path length or traffic overhead due to extra messaging packets are incurred.

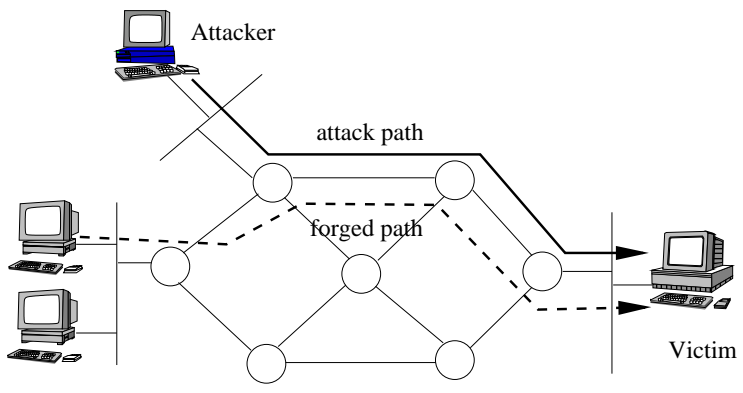

Fig. II.1. In PPM, an attacker can forge a path that is equally likely as the true attack path by transmitting corrupted packets that reach the victim untouched (i.e., unmarked).

Probabilistic packet marking [9], [13], [15] achieves the best of both worlds-space efficiency in the form of constant marking field and processing efficiency in the form of minimal router support - at the expense of introducing uncertainty due to probabilistic sampling of a flow's path. The latter has two important, and opposing, effects: (a) discovery of correct path information by sampling which aids the victim's objective of traceback, and (b) injection of corrupted information by the attacker. In the latter, with a certain probability a packet-however formatted by the attacker-will travel through untouched, which can impede the victim's ability to identify the true attack path. This is illustrated in Figure II.1. More generally, the number of forgeable paths that are from an information-theoretic point-of-view indistinguishable with respect to their validity from the true attack path can further render source identification difficult if their numbers are large. In [9], issue (a) was analyzed yielding a partial and, perhaps, overly optimistic evaluation of probabilistic packet marking as a DoS prevention method. The principal contribution of [9] lies in the investigation of coding issues aimed at further reducing the (constant) marking bits needed in the IP header via fragmentation. The IP option field is another possible candidate for implementing marking field coding. In this paper, we study the critical issue (b) - the attacker's ability to inject misleading information-and give a comprehensive analysis of the effectiveness of PPM under single-source and distributed DoS attacks, complemented by numerical evaluations.

We remark that PPM is not perfect and suffers under two additional weaknesses (they are not unique to PPM, however, and are shared by the other approaches). First, PPM is reactive in the sense that damage must occur before corrective actionsincluding source identification - can be undertaken by the victim. Second, PPM does not scale well under distributed DoS (DDoS) attacks in the sense that the more hosts an attacker is able to compromise and use as a distributed attack site, the greater the effort needed (approximately proportional) to identify the attack sites. Route-based distributed packet filtering [28] is a new approach which, in addition to matching the power of PPM, solves its weaknesses including the need to have a marking field.

\section{Probabilistic Packet Marking And Traceback}

\section{A. Network Model}

The network is given as a directed graph $G=(V, E)$ where $V$ is the set of nodes and $E$ is the set of edges. $V$ can be further partitioned into end systems (leaf nodes) and routers (internal nodes). The edges denote physical links between elements in $V$. Let $S \subset V$ denote the set of attackers and let $t \in V \backslash S$ denote the victim. We will first consider the case when $|S|=1$ (single-source attack) and treat the distributed DoS attack case separately. We assume that routes are fixed ${ }^{1}$, and

$$
\mathcal{A}=\left(s, v_{1}, v_{2}, \ldots, v_{d}, t\right)
$$

comprised of $d$ routers (or hops) $v_{1}, \ldots, v_{d}$, and of path length ${ }^{2}$ $d$ is called an attack path. A path $\mathcal{B}, \mathcal{B} \neq \mathcal{A}$, with destination node $t$ and source node $u(u \neq s)$ is called a forgeable path.

\section{B. Probabilistic Marking}

\section{B.1 Definition}

Let $N$ denote the number of packets sent from $s$ to $t$. We will leave the time duration or interval unspecified (typically $N \gg$ 1 and DoS attacks occur over a concentrated time period). A

\footnotetext{
${ }^{1}$ On the IP Internet, the majority of TCP sessions do not experience route changes during their connection lifetime. Generalization of PPM under dynamic routing (the routing process must be specified) is a problem for future work.

${ }^{2}$ Without loss of generality, we use a slightly modified definition of path length which counts the number of intermediate hops for notational convenience.
} 

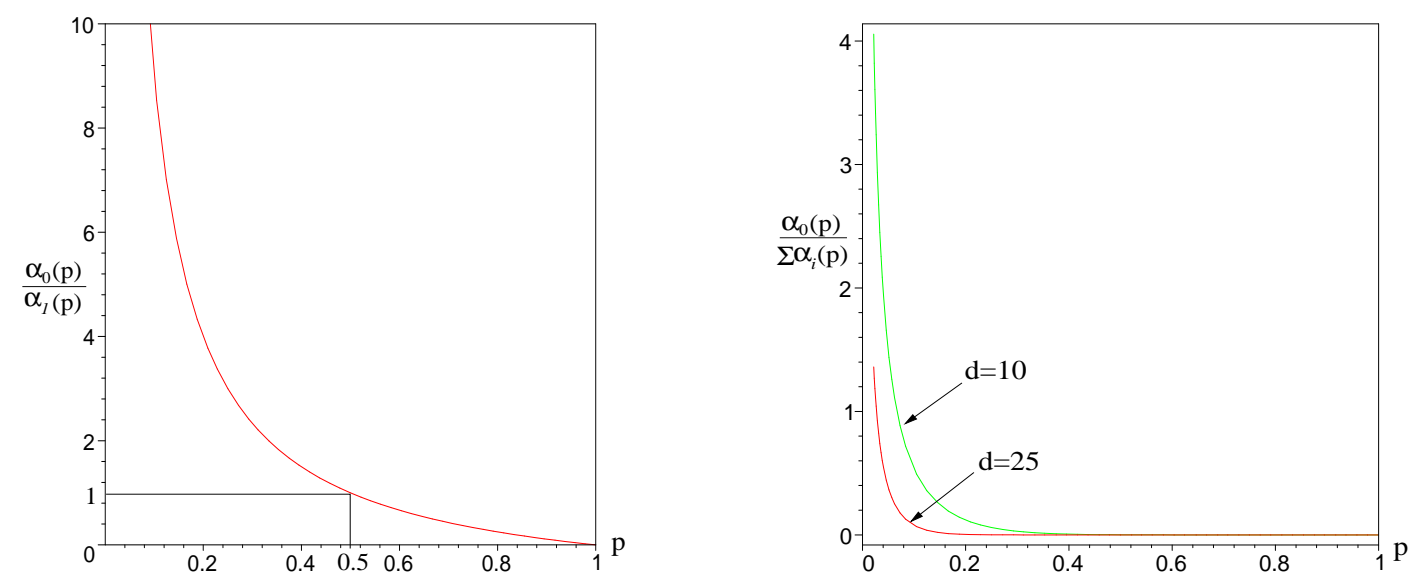

Fig. III.1. Left: $\alpha_{0}(p) / \alpha_{1}(p)$ as a function of $p$. Right: $\alpha_{0}(p) / \sum_{i} \alpha_{i}(p)$ as a function of $p$ for $d=10,25$.

packet $x$ is assumed to have a marking field where the identity of a link $\left(v, v^{\prime}\right) \in E$ traversed can be inscribed. A packet travels on the attack path $\mathcal{A}$ sequentially. At a hop $v_{i} \in\left\{v_{1}, \ldots, v_{d}\right\}$, packet $x$ is marked with the edge value $\left(v_{i-1}, v_{i}\right), i=1, \ldots, d$, with probability $p(0 \leq p \leq 1)$ where $v_{0}=s$. This process is called probabilistic marking. If a packet $x$ was already marked by a previous router, a new mark will replace or overwrite the old one. Let $x_{j}, j=0,1, \ldots, d$ denote the value of the marking field at node $v_{i}$. Let $X_{1}, X_{2}, \ldots, X_{d}$ be a set of i.i.d. binary random variables where $\operatorname{Pr}\left\{X_{i}=1\right\}=p, \operatorname{Pr}\left\{X_{i}=0\right\}=$ $1-p$, and $X_{i}=1$ indicates that marking was performed at node $v_{i} . x_{0}$ is under the control of the attacker who determines the initial marking value. Thus $x_{j}$ is a random variable depending on $X_{j}, X_{j-1}, \ldots, X_{1}$ and $x_{0}$, and we will be interested in the behavior of $x_{d}$.

\section{B.2 Path Sampling}

Let $\alpha_{i}(p)$ denote the probability that the arriving packet at the victim is lastly marked at node $v_{i}$ but nowhere after $v_{i}$. Thus

$$
\alpha_{i}(p)=\operatorname{Pr}\left\{x_{d}=\left(v_{i-1}, v_{i}\right)\right\}=p(1-p)^{d-i} .
$$

The probability that a packet sent from the attacker reaches the victim without being marked at any of the routers is $\alpha_{0}(p)=$ $(1-p)^{d}$. As with IP source address spoofing, the attacker may choose to inscribe a value $x_{0}$ which serves the purpose of hiding the attacker's identity. When $N$ packets are transmitted, the expected number of packets reaching target $t$ marked with the edge value $\left(v_{i-1}, v_{i}\right)$ is $n_{i}(p)=N \alpha_{i}(p)$. Note that

$$
\alpha_{1}(p) \leq \alpha_{2}(p) \leq \cdots \leq \alpha_{d}(p),
$$

and to receive a marked packet from $v_{1}$ containing the first link value $\left(s, v_{1}\right)$ requires $N \geq 1 / \alpha_{1}(p)$. Since $N$ (the attack volume) is a variable under the attacker's control, from a purely sampling point-of-view, edge $\left(s, v_{1}\right)$ is the "weakest link" requiring the most samples (i.e., packet transmissions) to recover the attack path. The expected number of samples needed to receive marked packets from all routers requires a logarithmic correction term, and is bounded above by (const $\cdot \ln d) / \alpha_{1}(p)$. This follows from the disjointness of $\alpha_{i}(p)$ and an application of the coupon collector's problem using the relaxed probability $\alpha_{1}(p)$, which yields the well-known solution $d \ln d+O(d)$. This has also been noted in [9].

\section{B.3 Marking Field Spoofing}

When $N$ packets are sent in the course of a DoS attack, the attacker can expect $n_{0}(p)=N(1-p)^{d}$ packets containing the attacker's inscribed value $x_{0}$ to reach the target untouched. By "corrupting" the marking field-in addition to spoofing the IP source address - the attacker may adversely impact the path reconstruction capability of the victim based on the $N$ packets received. The larger the fraction of corrupted marking field packets, the more damage the attacker can exact. What values to inscribe to achieve maximum effect is treated in the next section. With respect to the weakest point $v_{1}$, we are interested in the $p$ values for which

$$
\begin{aligned}
n_{0}(p) \geq n_{1}(p) & \Leftrightarrow \quad \alpha_{0}(p) \geq \alpha_{1}(p) \\
& \Leftrightarrow \quad(1-p)^{d} \geq p(1-p)^{d-1}
\end{aligned}
$$

which has the solution $p \leq 1 / 2$. That is, if $p \leq 1 / 2$ then spoofed packets will arrive more than true packets marked with the link value $\left(s, v_{1}\right)$. In general, we may consider the case

$$
\alpha_{0}(p) \geq \sum_{i=1}^{d} \alpha_{i}(p) \quad \Leftrightarrow \quad(1-p)^{d} \geq 1-(1-p)^{d}
$$

where the corrupted packets are in the absolute majority which holds for $p \leq 1-2^{-1 / d}$. For example, for $d=10$, the inequality holds if $p \leq 0.067$. Figure III.1 (left) shows the ratio $\alpha_{0}(p) / \alpha_{1}(p)$ as a function of $p$, and Figure III.1 (right) shows $\alpha_{0}(p) / \sum_{i} \alpha_{i}(p)$ as a function of $p$ for $d=10,25$.

Whereas $N, d$, and $x_{0}$ are under the attacker's control, the marking probability is a system parameter and, thus, the purview of the victim. The optimal selection of $N, d$, and $x_{0}$ by the attacker, and correspondingly optimal selection of $p$ by the victim to achieve their individual, conflicting objectives lies at the heart of the probabilistic PPM approach to source identification. In practice, we assume that an overall agreed-upon, effective $p$ value would be implemented at the routers. 


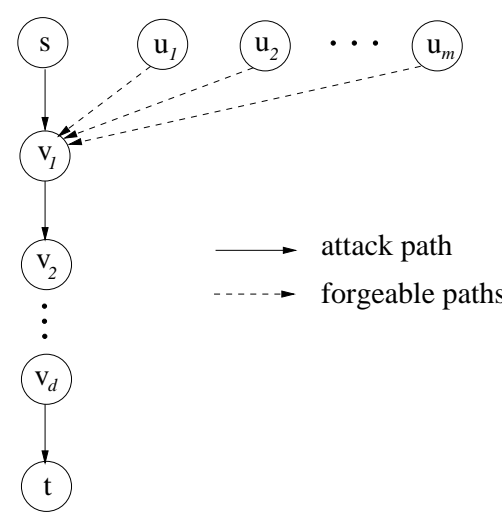

Fig. III.2. Attack path $\left(s, v_{1}, v_{2}, \ldots, v_{d}, t\right)$ and a set of $m$ forgeable paths $\left(u_{i}, v_{1}, \ldots, v_{d}, t\right), i=1, \ldots, m$, joined at $v_{1}$.

\section{Traceback Problem}

Consider an attacker with attack path $\mathcal{A}=\left(s, v_{1}, \ldots, v_{d}, t\right)$ and forgeable paths $\mathcal{B}_{i}=\left(u_{i}, v_{1}, \ldots, v_{d}, t\right), i=1, \ldots, m$, at distance $d$, joined at $v_{1}$ forming the (caterpillar) subgraph shown in Figure III.2. This particular attack pattern is of interest (i) because it targets the "weakest" point of probable path recovery by the victim according to (III.1), (ii) attacker $s$ can generate packets that, unless marked at $v_{1}$, will be indistinguishable from real packets originating at $u_{i}$ and arriving at $t$, (iii) other attack configurations can be analyzed using the tools developed for the caterpillar subgraph, and (iv) the concepts underlying optimal decision making by both attacker and victim are easily brought out. The traceback problem in a caterpillar graph is a special case of the traceback problem in general topologies, which is discussed in the full paper [1]. One of the three decision variables-the attacker's marking field spoof variable $x_{0}$ - can be fixed by the following informationtheoretic argument. Let $n_{i}^{s}(p)$ be the number of spoofed packets arriving at $t$ with the marking field containing $\left(u_{i}, v_{1}\right)$. Assume $n_{0}(p)=\sum_{i=1}^{m} n_{i}^{s}(p)$. That is, all packets transmitted by the attacker are inscribed with spoofing values from the link set $\left\{\left(u_{i}, v_{1}\right): i=1,2, \ldots, m\right\}$. If it holds that

$$
n_{1}(p)=n_{1}^{s}(p)=n_{2}^{s}(p)=\cdots=n_{m}^{s}(p),
$$

then by (ii) all $m+1$ paths are equally likely-i.e., the attack could have been undertaken from any of the nodes $s, u_{1}, u_{2}, \ldots, u_{m}$ yielding the same outcome in terms of collected marking values at $t$. Of course, by the probabilistic nature of the marking process, exact equality cannot be expected to hold. Instead, if the marginal densities can be equated

$$
\alpha_{1}(p)=\alpha_{1}^{s}(p)=\alpha_{2}^{s}(p)=\cdots=\alpha_{m}^{s}(p),
$$

entropy is maximal, and by symmetry, each of the nodes $\left\{s, u_{1}, u_{2}, \ldots, u_{m}\right\}$ is an equally likely candidate. We will call $m$ - a function of $p$ and spoofing variable $x_{0}$ - the uncertainty factor with respect to marking probability $p$. For a formal definition of the "indistinguishability notion," we refer the reader to [1]. In the context of traceback, the uncertainty factor $m$ is the objective function for measuring the effectiveness of traceback. The larger $m$ is, the more the processing cost incurred by the victim to trace back the attack source. Thus, the objective of the attacker is to maximize $m$, whereas the objective of the victim is to minimize $m$. A minimax optimization problem for the attacker and victim can be formulated as follows:

$$
\begin{gathered}
\min _{p} \max _{x_{0}} m\left(p, x_{0}\right) \\
\text { subject to (III.4) }
\end{gathered}
$$

where the maximum is over all distributions of $x_{0}$ viewed as a random variable. The minimax formulation biases toward the victim. The formulation in (III.5) does not incorporate the attack volume $N$ and thus unduly favors the victim. A sampling constraint is added by requiring

$$
N \alpha_{1}(p)=N p(1-p)^{d-1} \geq 1 \text {. }
$$

Thus the refined minimax optimization reflecting the victim's sampling constraint is given by

$$
\begin{gathered}
\min _{p} \max _{x_{0}, N} m\left(p, x_{0}\right) \\
\text { subject to (III.4) and (III.6). }
\end{gathered}
$$

Note that $N$ is incorporated as part of the attacker's decision variable due to constraint (III.6). $N \alpha_{1}(p)$ as a function of $p$ has a unimodal (or bell) shape with peak at $p=1 / d$. Thus decreasing $N$ can shrink the size of the feasible region defined by (III.6).

\section{Analysis of Single-Source DoS Attack}

This section analyzes PPM under single-source DoS attacks. We first derive performance bounds for the minimax optimization problem, and then give numerical evaluations using Internet related parameters that complement the analytical results.

\section{A. Minimax Optimization}

A necessary condition for (III.4) to hold is that when transmitting a packet, the attacker inscribes spoofed link values with uniform probability, i.e.,

$$
\operatorname{Pr}\left\{x_{0}=\left(u_{i}, v_{1}\right)\right\}=\frac{1}{m}, \quad i=1,2, \ldots, m .
$$

Condition (IV.1) can be further derandomized-i.e., replaced by a deterministic procedure that emulates uniform generation-if information contained in the sequential arrival of marked/spoofed packets is not considered. In conjunction with (IV.1), a necessary and sufficient condition for (III.4) is

$$
\begin{aligned}
m \alpha_{1}(p)=\alpha_{0}(p) & \Leftrightarrow \quad m p(1-p)^{d-1}=(1-p)^{d} \\
& \Leftrightarrow \quad m=\frac{1}{p}-1
\end{aligned}
$$

That is, given $p$ (determined by the victim), the attacker can achieve an uncertainty factor of $m=(1 / p)-1$. Thus $m=$ $(1 / p)-1$ is the maximal uncertainty factor satisfying (III.4) for a given $p$. Without the sampling constraint (III.6), the victim can affect

$$
\inf _{p}\left(\frac{1}{p}-1\right)=0
$$



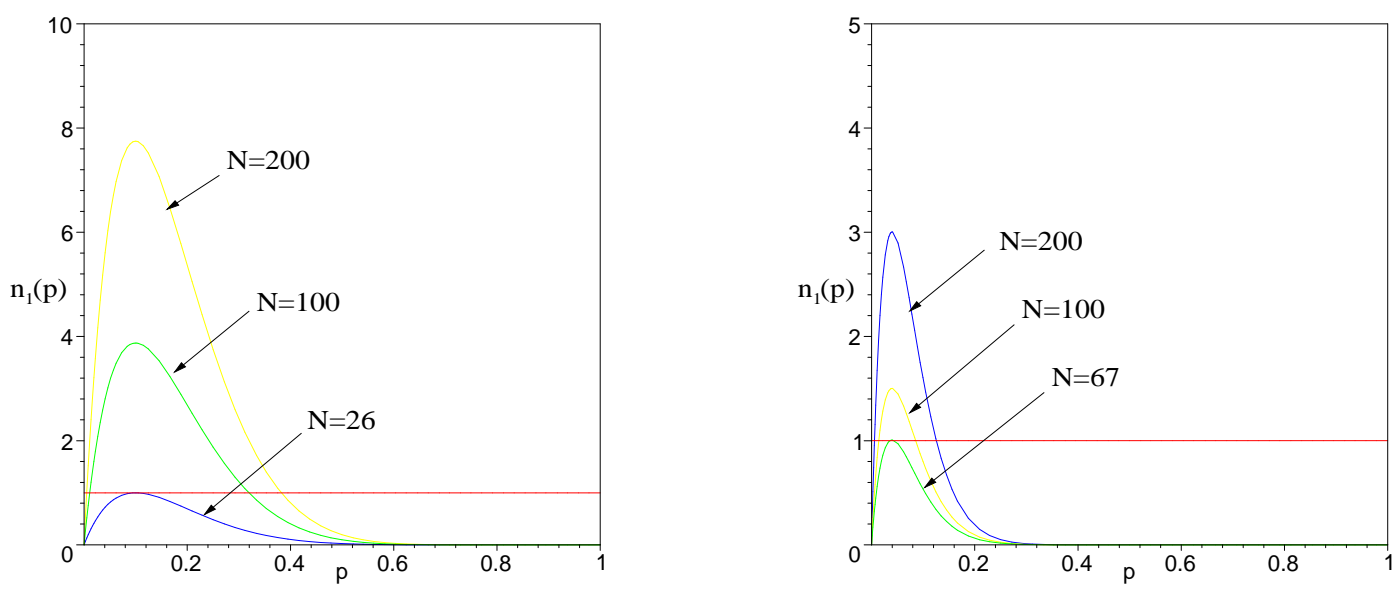

Fig. IV.1. Left: $n_{1}(p)$ as a function of $p$ for $N=26,100$, and 200 when $d=10$. Right: Corresponding plot when $d=25$.

since $0 \leq p<1$. Since $p=1$ is disallowed-necessary for probabilistic path discovery when the hop count $d$ is at least 2we have

$$
m=(1 / p)-1 \searrow 0 \text { as } p \nearrow 1 .
$$

The uncertainty factor achievable by the attacker becomes null since, $m$ being an integer representing the in-degree of router $v_{1}$, only $\lfloor m\rfloor$ matters. With the sampling constraint (III.6) constraining the victim from choosing $p$ arbitrarily close to 1 , we need to compute the min-max over the feasible region

$$
L=\left\{(p, N): N p(1-p)^{d-1} \geq 1\right\}
$$

defined by (III.6) where $L$ is parameterized by the attack distance $d$. It can be checked that for all $d \geq 2, L$ is convex in $p$. Thus the feasible region $L$ defined by both the attacker and victim's moves is a union of convex sets $L_{N}$ (the set $L$ keeping the second coordinate fixed at $N$ ) for $N \geq N_{0}$ where $N_{0}=N_{0}(d)$ is the least number-a function of $d$-such that $\left(p, N_{0}\right) \in L$ for some $p$.

Theorem 1: For all $d \geq 2, L_{N}$ is convex. Furthermore, $L_{N^{\prime}} \supseteq L_{N}$ if $N^{\prime} \geq N$.

Theorem 1 shows that the minimax problem can be viewed as a sequence of convex minimization problems of the objective function $(1 / p)-1$ over $L_{N}$ for $N=N_{0}, N_{0}+1, \ldots$ Thus there is a unique solution. The next result gives a performance bound on the attacker's ability to hide his identity under PPM.

Theorem 2: Let $m^{*}$ be the solution of the constrained minimax problem given by (III.7). Then $m^{*} \leq d-1$.

Theorem 2 shows that the maximum achievable uncertainty factor-i.e., equally likely forged paths-cannot exceed $d-1$, the distance between the attacker and victim. Thus the farther the attack site from the target, the more uncertainty can be injected. On the Internet [29], most path lengths are bounded by 25 , and thus this puts an upper bound on the effectiveness of single-source DoS attacks when subject to probabilistic packet marking. An immediate consequence of Theorem 2 is the following corollary which shows that $d-1$ can be tight.
Corollary 1: If $N=d^{d} /(d-1)^{d-1}$ then $m^{*}=d-1$.

Thus the attacker, by judiciously choosing the attack volume, can maximally hide his identity given by $d-1$. Since $d^{d} /(d-$ $1)^{d-1} \propto d$, this occurs at a drastic cost in reduced attack volume which may fail to affect significant "denial of service" at the target, thus taking the bite out of the attack.

\section{B. Approximation of Uncertainty Factor}

To find a feasible region of $p$ for $N p(1-p)^{d-1} \geq 1$, we need to solve the equation $N p(1-p)^{d-1}=1$. This equation is transformed to the polynomial $x^{n}-x^{n-1}+c$ by substitution of $p, N, d$ with $1-x, 1 / c, n$, respectively. It is not possible, however, to factor the polynomial with $c=1 / N$ to find its roots. Also, there are no known formulae for the roots of polynomials with degree $n \geq 5$ [30]. Therefore, we derive approximate solutions for the minimax optimization problem in addition to the qualitative results derived in the previous section.

Without loss of generality, we divide $N p(1-p)^{d-1}=1$ by $N$, and represent $p$ as $1-x(0 \leq x \leq 1)$. Thus, the equation becomes

$$
(1-x) x^{d-1}=\frac{1}{N} \text {. }
$$

Assuming $N \gg 1$ which is justified by $N$ denoting the attack volume of DoS, the right-hand-side becomes $\frac{1}{N} \approx 0$. Thus, the solution is close to 0 or 1 . First, consider the case where a root is close to 1 . The exponential term will be close to 1 , yielding the approximate solution $x=1-1 / N$. For this value of $x$, the exponential term on the left-hand-side becomes $(1-1 / N)^{d-1}$. This term approaches 1 as $N \rightarrow \infty$. For example, its value is 0.99976 when $N=10^{5}$ and $d=25$, which is small compared to unity. Thus, we arrive at an approximation to the root.

Next, consider the case when the root is close to 0 . The term $(1-x)$ will be close to 1 , and may be neglected. The equation $x^{d-1}=1 / N$ gives an approximate solution $x=\frac{1}{N}^{\frac{1}{d-1}}$. The value of $x=\frac{1}{N}^{\frac{1}{d-1}}$ is close to 0 for large $N$ so that $(1-x) \approx 1$. Thus, since $x$ is approximately $1-(1 / N)$ or $\frac{1}{N}^{\frac{1}{d-1}}$, the corresponding $p$ is $1 / N$ or $1-\frac{1}{N}^{\frac{1}{d-1}}$. Therefore, $p$ is approximately in the following region for satisfying the sampling constraint 

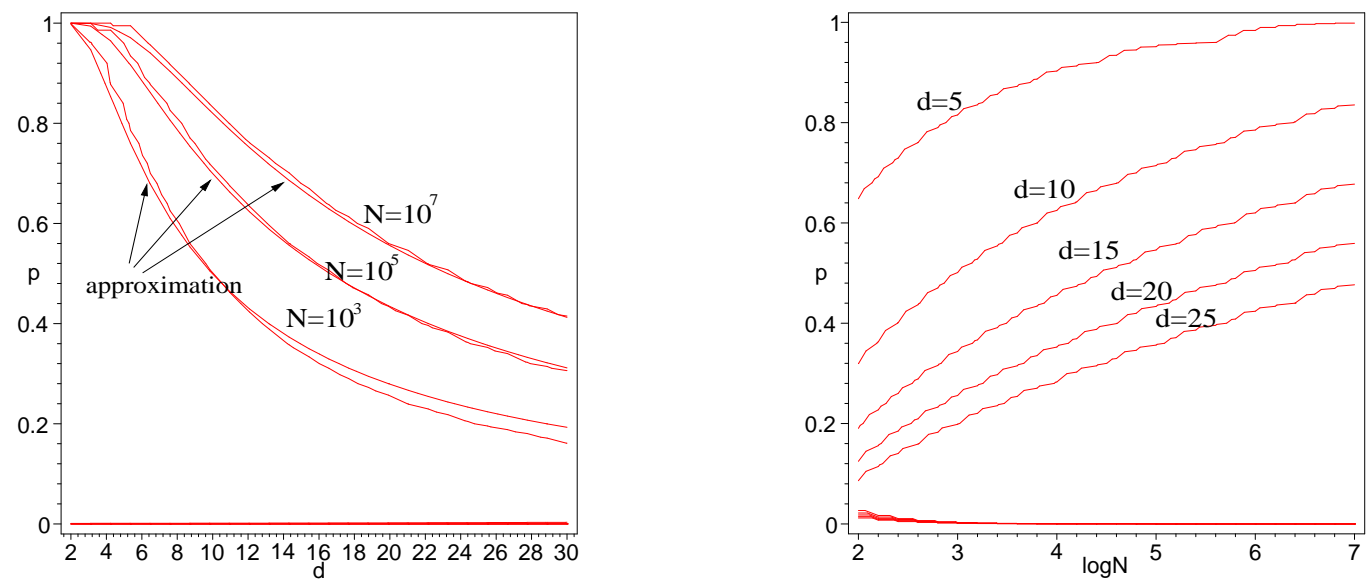

Fig. IV.2. Left: Upper bound of marking probability $p$ and its approximation as a function of $d$ for $N=10^{3}, 10^{5}, 10^{7}$. Right: Upper bound of $p$ as a function of $N$ (i.e., its logarithm) for $d=5,10,15,20$, and 25 .

$N p(1-p)^{d-1} \geq 1$

$$
\frac{1}{N} \leq p \leq 1-\left(\frac{1}{N}\right)^{\frac{1}{d-1}} .
$$

Hence, the maximum uncertainty value $m$ of the min-max optimization problem is given by

$$
m \approx \frac{N^{-\frac{1}{d-1}}}{1-N^{-\frac{1}{d-1}}} .
$$

When $N=10^{5}$ and $d=25$, the uncertainty factor $m$ is approximated by 1.6247 . When $N=10^{7}$ and $d=25, m$ is further reduced to 1.0446. From the approximate analysis of the maximally attainable uncertainty factor, we conclude that choosing a maximum allowable $p$ by a victim results in the limited ability of an attacker to hide his identity (e.g., $m=1 \sim 2$ when $N=10^{5} \sim 10^{7}$ ).

\section{Numerical Evaluation}

In this section, we give numerical solutions to (III.7) that complement the bounding results and the approximate solutions given in the previous sections.

\section{C.1 Marking Probability}

Probabilistic marking with respect to its encoding using the IP header's fragmentation field can be efficiently implemented using code distribution over multiple packets. We refer the reader to [9] for a discussion.

First we measure the range of $p$ which satisfies $n_{1}(p) \geq 1$ for different values of $N$ and $d$. Figure IV.1 (left) shows $n_{1}(p)=$ $p(1-p)^{d-1} N$ as a function of $p$ for $N=26,100$, and 200 when $d=10$. The allowable range of $p$ (i.e., the set $L_{N}$ ) is the region where values of $n_{1}(p)$ become larger than 1 . This can be discerned by the intersection of $n_{1}(p)$ with the constant line 1 . For this graph, the upper bound of $p$ is minimized at $1 / d=0.1$ with $N=10^{10} / 9^{9} \approx 26$. As $N$ decreases, the upper bound of $p$ decreases until $N$ reaches to $d^{d} /(d-1)^{d-1}$. Figure IV.1 (right) shows the corresponding graphs when $d=25$.
Figure IV.2 (left) shows the feasible range of $p$ as a function of $d$ when $N=10^{3}, 10^{5}, 10^{7}$, and their approximations. The plots show that our approximation is close to the solution. In particular, as $N$ increases, the approximation becomes tighter, especially, for $d$ large. The upper graphs represent the upper bounds of $p$ which correspond to the minimax solution of (III.7), and the bottom graphs are of the feasible region $L_{N}$ which are near zero. We observe that as $d$ increases, the upper bound of $p$ decreases. Since the Internet has a bounded diameter, the upper bound of $p$ stays at "high" values yielding uncertainty factors $m=(1 / p)-1$ that are commensurately "low." Figure IV.2 (right) shows the minimax solution as a function of traffic volume $N$ (i.e., its $\log a r i t h m \log N$ ) for $N$ in the range $100 \sim 10^{7}$ when $d=5,10,15,20$, and 25 . We observe that to reduce the minimax value of $p$ and thus increase the uncertainty factor $m$, the attack volume needs to be decreased exponentially which is a high penalty to pay in a DoS attack.

\section{C.2 Attack Distance}

Let us consider the range of forgeable paths when $d=25$, since few paths on the Internet exceed that distance [29]. In the case of $N=10^{5}$, the marking probability $p$ must be in the range $0.1 \times 10^{-4}<p<0.3536$ to satisfy the sampling constraint. For this range, the number of forgeable paths $m$ is shown in Figure IV.3 (left). While the uncertainty factor $m$ lies in the range $1<m<10^{5}$, a victim can reduce $m$ to 2 by choosing the maximal feasible $p$, i.e., $p=0.35$. When we increase $N$ to $10^{7}, p$ is in the range of $0.1 \times 10^{-6}<p<0.4729$, and its corresponding value of $m$ is shown in Figure IV.3 (left). From the above instances, we observe that even though PPM cannot pinpoint the attack host's location, the number of possible candidates is a manageable constant which can help facilitate on-line traceback and increase the deterrent factor.

Let us consider the effect of the attacker's location to the traceback. As shown in Figure IV.2, as $d$ increases, the upper bound of $p$ decreases, which increases the uncertainty factor $m$. Given $N$, as distance $d$ decreases, the expected number of spoofed packets, $N_{s}$, will increase for any given value of $p$. We note, however, that the ability of an attacker to hide the attack 

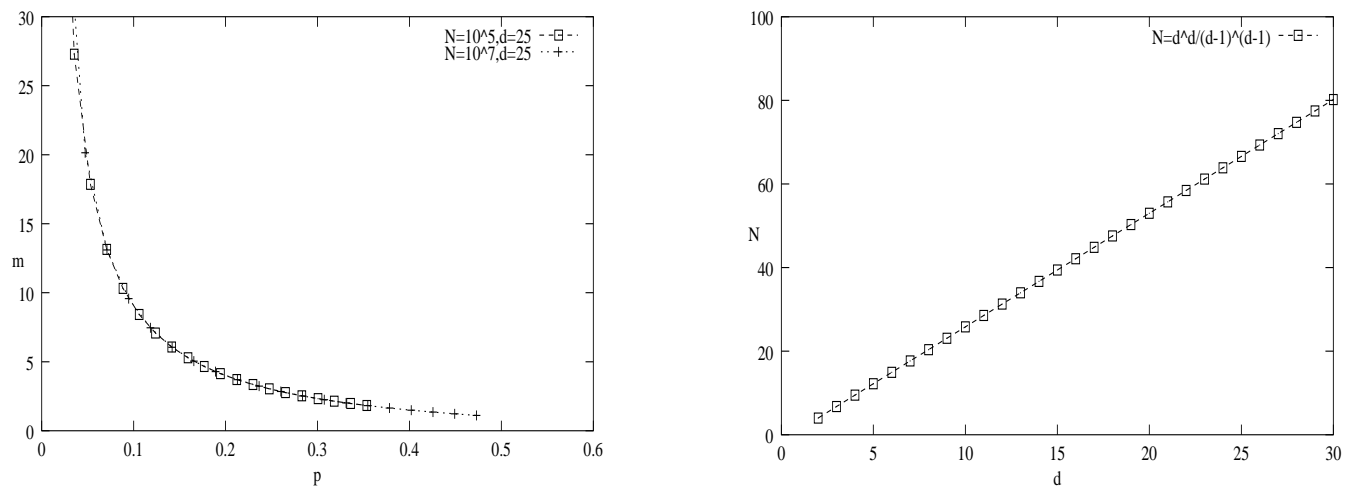

Fig. IV.3. Left: The lower bound of forgeable paths $m$ as a function of $p$ for $d=25$ and $N=10^{5}, 10^{7}$. Right: The least attack volume $N$ needed to satisfy the sampling constraint (III.6).

location is not in proportion to the number of spoofed packets received by the victim. Conversely, as $d$ increases, while the number of spoofed packets received by a victim decreases, the uncertainty factor $m$ increases. Therefore, when the source of an attack is far from the victim, the attacker becomes more potent at impeding traceback. Since the distance between an attacker and victim is bounded on the Internet, an attacker has limited ability to hide his location when subject to probabilistic packet marking.

\section{C.3 Attack Volume}

For the purpose of path reconstruction on the victim side, $N$ needs to be at least $d^{d} /(d-1)^{d-1}$ to satisfy the sampling constraint. Figure IV.3 (right) shows the sampling lower bound on $N$ when $d=2 \sim 30$. As $N$ increases, the victim can reduce the number of forgeable paths to less than $d-1$. Therefore, if an attacker transmits a small number of packets near the sampling lower bound, the victim will additionally suffer under a sampling problem. This points toward the fact that amplified confusion can be achieved by mounting distributed DoS attacks where each attack host contributes a small fraction of the total attack volume.

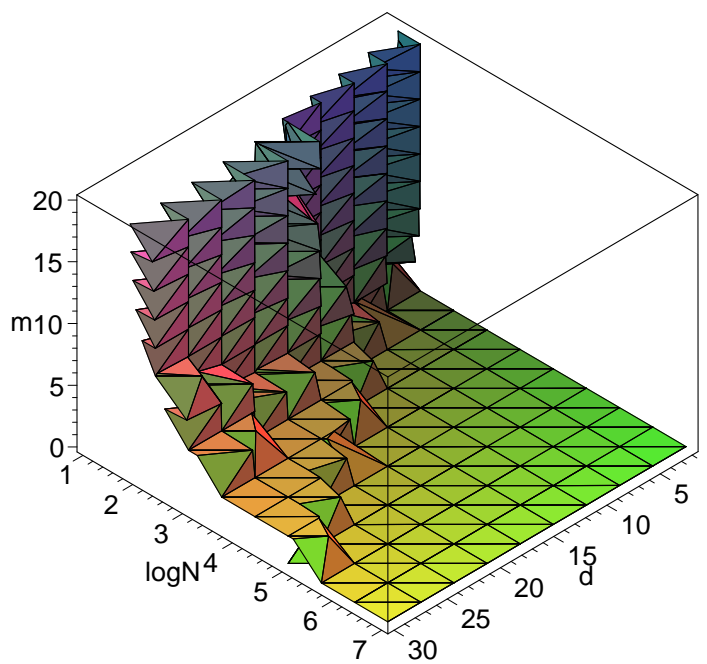

Fig. IV.4. The minimax solution of forgeable paths $m$ as a function of $N$ (shown for $\log N$ ) and $d$.
Figure IV.4 shows the minimax solution of uncertainty factor $m$ as a joint function of $d$ and $\log N$. The value of $m$ is plotted for $10^{1} \leq N \leq 10^{7}$ and $2 \leq d \leq 30$. As noted earlier, an increase in $d$ leads to an increase in $m$. Whereas the impact of $d$ is gradual - in fact, linear (i.e., upper bounded by $d-1$ ), the impact of $N$ is more pronounced. With a small attack volume, e.g., $N=10 \sim 100$, an attacker can keep the victim at an uncertainty level approaching 20 . As $N$ increases to $N=10^{3} \sim$ $10^{5}$, however, $m$ can achieve values only in the range $1 \sim 4$ even at $d=25$. This means that a victim can effectively localize the physical source of an attack to $2 \sim 5$ candidates. This makes it intrinsically difficult for a DoS attacker to wreck havoc using single-source attacks when PPM is employed by the network to facilitate traceback. Of course, it is unrealistic to assume that $p$ can be programmed by different users to suit their individual needs. The small constant upper bound on $m$ admits the policy of setting $p$-once and for all—for a sufficiently large distance $d$ and conservative attack volume $N$ which renders single-source traceback practically feasible.

\section{Distributed DoS ATtack}

\section{A. Key Issues}

Given the theoretically and practically bounded impact of single-source DoS attack under probabilistic packet marking, distributed DoS attacks present a potentially important dimension to the source identification problem. In Section IV we showed that the uncertainty factor in single-source attack can be amplified up to 20 if the path length is sufficiently large, however, this occurs at the cost of drastic-i.e., exponentialreduction in traffic volume (cf. Figure IV.4) which may render the attack ineffective with respect to achieving "denial of service." Attack volume may be recovered by mounting concurrent, small volume attacks from a number of sites, but its efficiency needs to be evaluated with respect to the cost of mounting distributed attacks which grows with the number of hosts engaged in the attack. In particular, following the uncertainty optimization framework - minimization for the victim and maximization for the attacker - of Section IV, given a desired attack volume $N$, an amplification factor of $M$ can be trivially achieved by mounting $N / M$-volume attacks from $M$ separate attack sites. That is, even in the absence of forging or spoofing of the marking field, the victim will need to process $M$ total 

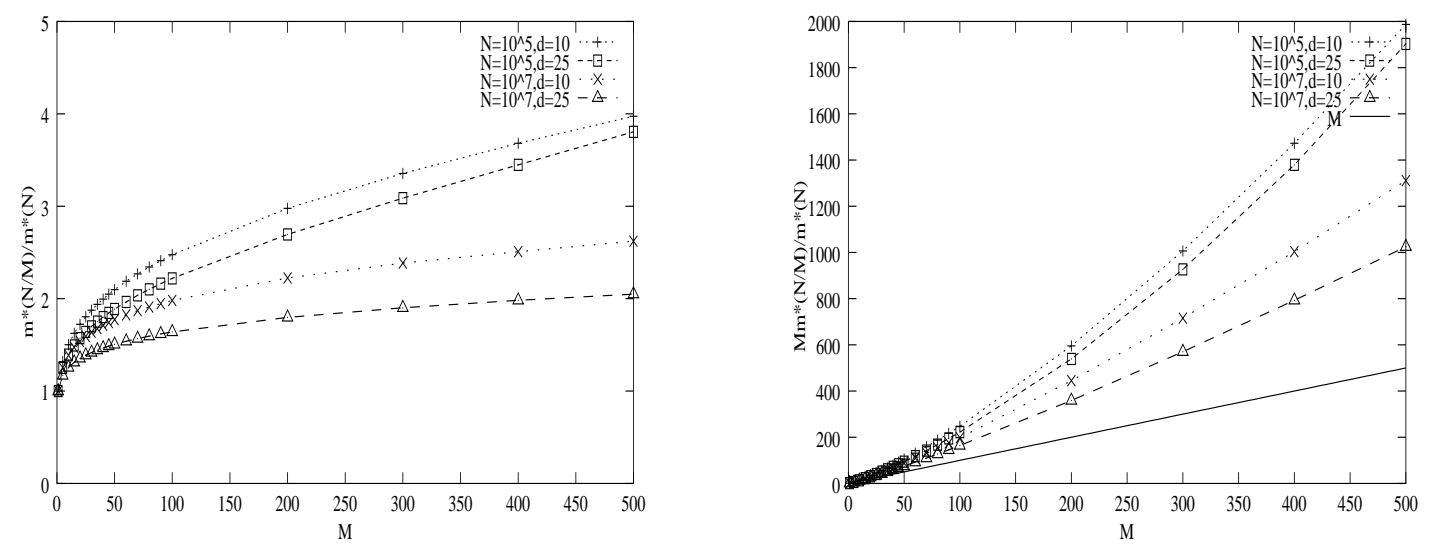

Fig. V.1. Left: The coefficient of expansion $\frac{m^{*}(N / M)}{m^{*}(N)}$ to the uncertainty amplification measured in the range $M=1 \sim 500$. Right: The uncertainty factor $\frac{M m^{*}(N / M)}{m^{*}(N)}$ measured in the range $M=1 \sim 500$.

attack paths when fielding a defense, on-line or off-line. With spoofing of the marking field, amplification may be greater. In fact, it is given by the expression $M\left(m^{*}(N / M) / m^{*}(N)\right)$ where $m^{*}(\cdot)$ is a function depicting the optimum (i.e., minimax) uncertainty factor for the traffic volume given in the argument. Without spoofing, $m^{*}(N / M)=m^{*}(N)$, and we arrive at the amplification factor $M$. With spoofing, $m^{*}(N / M) \geq m^{*}(N)$.

\section{B. Distributed DoS Attack Model}

\section{B.1 Classification}

The traceback problem in distributed DoS attacks can be classified into two categories in accordance with the objectives underlying the attack and its susceptibility to traceback. In anysource traceback, the attacker is assumed to be vulnerable to further traceback once a compromised attack host is identified (e.g., due to state information left on the host). Thus the attacker seeks to fortify the weakest link-i.e., maximize the uncertainty factor of each individual attack host-whereas the victim tries to find a weak attack host. In all-source traceback, we assume the attacker is able to mount stateless intrusions when gathering attack hosts, and thus his objective is to maximize total uncertainty (vs. individual uncertainty in the any-source traceback case) since quick traceback of individual attack hosts does not present a danger with respect to revealing traceback information. The attacker's objective is to maximize the number of forged paths that the victim has to process, and the victim's goal is to isolate or shut down traffic flow emanating from comprised hosts.

\section{B.2 Traceback Analysis}

An environment for distributed DoS attack is described as follows. Given $M$ distinct sources, each source $s_{i}$ sends $N_{i}$ packets to victim $t$ at $d_{i}$ distance for $1 \leq i \leq M$. An attack path $\mathcal{A}_{i}$ is represented by $\mathcal{A}_{i}=\left(s_{i}, v_{i, 1}, v_{i, 2}, . ., v_{i, d_{i}}, t\right)$. Without loss of generality, assume $d_{i} \leq d_{j}$ for $i<j$. The expected number of spoofed packets received by the victim from attack host $s_{i}$ is

$$
N_{s_{i}}=\alpha_{i, 0}(p) N_{i}=(1-p)^{d_{i}} N_{i}
$$

for $1 \leq i \leq M$. The expected number of packets marked by $v_{i, 1}$ is

$$
n_{i, 1}(p)=\alpha_{i, 1}(p) N_{i}=p(1-p)^{d_{i}-1} N_{i} .
$$

An attack host may use $N_{s_{i}}$ to increase its uncertainty factor $m^{i}$, or it may use its forged packets to help amplify the uncertainty factor $m^{j}$ of some other attack host $j \neq i$. That is, the attack hosts, in a distributed DoS attack, may engage in cooperative actions to achieve a common objective.

In the case of any-source traceback, the objective of the attacker is to maximize

$$
\min _{1 \leq i \leq M} m^{i}
$$

which is tantamount to fortifying the weakest attack host with respect to its uncertainty factor. Thus, (V.1) yields

$$
\min _{1 \leq i \leq M}\left\{\frac{\alpha_{i, 0}(p)}{\alpha_{i, 1}(p)}\right\}=\min _{1 \leq i \leq M}\left\{\frac{(1-p)^{d_{i}}}{p(1-p)^{d_{i}-1}}\right\}=\frac{1}{p}-1 .
$$

Thus, the any-source traceback case reduces to the single-source traceback problem as affected by the definition.

In the case of all-source traceback, the objective of the attacker lies in maximizing

$$
m=\sum_{i=1}^{M} m^{i}
$$

To affect an increase in $m$, an attack host may send spoofed packets whose aim is to amplify another attack host's uncertainty factor rather than its own. The objective function can be further simplified

$$
\sum_{i=1}^{M} m^{i}=\sum_{i=1}^{M} \frac{\alpha_{i, 0}(p)}{\alpha_{i, 1}(p)}=\sum_{i=1}^{M} \frac{(1-p)^{d_{i}}}{p(1-p)^{d_{i}-1}}=M\left(\frac{1}{p}-1\right)
$$

due to its lack of dependence on $N_{i}$ and $d_{i}$. Thus the derivation shows that from the attacker's point-of-view, one way of maximizing $m=\sum_{i=1}^{M} m^{i}$ is to perform $M$ separate maximizations 
on each attack host. As with the any-source traceback case, allsource traceback reduces to the single-source traceback problem and does not necessitate cooperation among the attack hosts to achieve maximum uncertainty amplification. When performing the constrained minimax optimization (III.7) on each attack host as given by the single-source formulation in Section III, $d_{i}$ and $N_{i}$ only enter in the $M$ constraints corresponding to (III.6).

\section{Numerical Evaluation of Traceback}

To measure the (in)effectiveness of traceback in a distributed DoS attack setting, we perform comparative evaluation with single-source attack where the total traffic volume is held constant. As discussed in Section V-A, our aim lies in evaluating the degree to which distributed DoS attack under probabilistic packet marking can achieve uncertainty amplification above and beyond the distribution factor $M$ achievable in the trivial case.

Let $N$ be the total attack traffic volume - the same for singlesource attack as well as distributed DoS attack-and let $N_{i}=$ $N / M, d_{i}=d, 1 \leq i \leq M$, which facilitates comparability. Let $m^{*}\left(N_{i}\right)$ be the uncertainty factor achievable by $N_{i}$. Then the ratio $m^{*}(N / M) / m^{*}(N)$ represents the expansion rate to uncertainty factor with respect to the distribution factor $M$. Figure V.1 (left) shows the coefficient of expansion to uncertainty amplification in an $M$-distributed attack where $M=1 \sim 500$. As $M$ increases, the coefficient of expansion increases, and achieves higher gains with small $N$ and small $d$. This implies that traceback to a single source becomes more difficult as the attack volume is scattered into smaller units.

Figure V.1 (right) shows the amplification factor $\frac{M m^{*}(N / M)}{m^{*}(N)}$ as a function of $M$. The larger $M$, the higher the amplification. Thus, given an attack volume, as attack sources are distributed, the uncertainty injected into traceback can be amplified beyond the effect afforded by distribution.

\section{CONCLUSION}

Recently probabilistic packet marking has been proposed for tracing the source-i.e., origin — of an DoS attack. While PPM has the advantages of efficiency and implementability over deterministic packet marking and router based logging/messaging, it has the potential drawback that an attacker may impede traceback by sending packets with spoofed marking field values as well as spoofed source IP addresses. This paper analyzed the effectiveness of PPM in a minimax adversarial context where the attacker is allowed to spoof the marking field to achieve maximum confusion at the victim. Our analysis shows that, while it is always possible for an attacker to impede exact traceback by the victim, the attacker's ability to affect uncertainty is limited in internetworks with bounded diameters similar to the Internet, when a suitable marking probability is chosen. Thus, for singlesource attacks PPM is effective at localizing the attack origin. In a distributed DoS attack, however, as the number of attack sources mounted increases, traceback is rendered more difficult due to an uncertainty amplification effect above and beyond the distribution factor $M$.

\section{REFERENCES}

[1] K. Park and H. Lee, "On the effectiveness of probabilistic packet marking for IP traceback under denial of service attack,” Tech. Rep. CSD-00-013,
Department of Computer Sciences, Purdue University, June 2000.

[2] Lee Garber, "Denial-of-service attacks rip the Internet," Computer, pp. 12-17, Apr. 2000.

[3] John Elliott, "Distributed denial of service attack and the zombie ant effect," IT Professional, pp. 55-57, March/April 2000.

[4] Jari Hautio and Tom Weckstrom, "Denial of service attacks," Mar. 1999, http://www.hut.fi/u/tweckstr/hakkeri/DoS_paper.html.

[5] John D. Howard, An Analysis of Security Incidents on the Internet, Ph.D. thesis, Carnegie Mellon Univerisity, Aug. 1998.

[6] NightAxis and Rain Forest Puppy, "Purgatory 101: Learning to cope with the SYNs of the Internet," 2000, Some practical approaches to introducing accountability and responsibility on the public internet, http://packetstorm.securify.com/papers/contest/RFP.doc.

[7] Computer Emergency Response Team, "Denial of service," Feb. 1999, Tech Tips, http://www.cert.org/tech_tips/denial_of_service.html.

[8] Computer Emergency Response Team (CERT), "CERT Advisory CA-2000-01 Denial-of-service developments,” Jan. 2000, http://www.cert.org/advisories/CA-2000-01.html.

[9] Stefan Savage, David Wetherall, Anna Karlin, and Tom Anderson, "Practical network support for IP traceback," in Proc. of ACM SIGCOMM, Aug. 2000, pp. 295-306.

[10] Steven M. Bellovin, "ICMP traceback messages," Mar. 2000, Internet Draft: draft-bellovin-itrace-00.txt (expires September 2000).

[11] Cisco Systems, "Characterizing and tracing packet floods using Cisco routers," Aug 1999.

[12] Glenn Sager, "Security fun with OCxmon and cflowd," Nov. 1998, Presentation at the Internet 2 Working Group.

[13] Dawn Song and Adrian Perrig, "Advanced and authenticated marking schemes for IP traceback," Tech. Rep. UCB/CSD-00-1107, Computer Science Department, University of California, Berkeley, 2000.

[14] Robert Stone, "Centertrack: An IP overlay network for tracking DoS floods," in Proc. of 9th USENIX Security Symposium, Aug. 2000.

[15] H. Burch and B. Cheswick, "Tracing anonymous packets to their approximate source," Dec. 1999, Unpublished manuscript.

[16] David Clark, "The design philosophy of the DARPA internet protocols," in Proc. SIGCOMM '88, 1988.

[17] Robert T. Morris, "A weakness in the 4.2BSD Unix TCP/IP software," Tech. Rep. Computer Science \#117, AT\&T Bell Labs, Feb. 1985.

[18] Gaurav Banga, Peter Druschel, and Jeffrey C. Mogul, "Resource containers: A new facility for resource management in server systems," in Proc. of the third USENIX/ACM Symp. on Operating Systems Design and Implementation (OSDI'99), Feb. 1999, pp. 45-58.

[19] Catherine Meadows, "A formal framework and evaluation method for network denial of service," in Proc. of the 1999 IEEE Computer Security Foundations Workshop, June 1999.

[20] Christoph L. Schuba, Ivan V. Krsul, Markus G. Kuhn, Eugene H. Spafford, Aurobindo Sundaram, and Diego Zamboni, "Analysis of a denial of service attack on TCP," in Proc. of the 1997 IEEE Symp. on Security and Privacy, May 1997, pp. 208-223.

[21] Oliver Spatscheck and Larry L. Peterson, "Defending against denial of service attacks in Scout," in Proc. of the third USENIX/ACM Symp. on Operating Systems Design and Implementation (OSDI'99), Feb. 1999, pp. 59-72.

[22] CERT/CC, SANS Institute, and CERIAS, "Consensus roadmap for defeating distributed denial of service attacks," Feb. 2000, A Project of the Partnership for Critical Infrastructure Security, http://www.sans.org/ddos_roadmap.htm.

[23] Paul Ferguson and Daniel Senie, "Network ingress filtering: Defeating denial of service attacks which employ IP source address spoofing," May 2000, RFC 2827.

[24] Daniel Senie, "Changing the default for directed broadcasts in routers," Aug. 1999, RFC 2644.

[25] Computer Emergency Response Team (CERT), "Results of the distributed-systems intruder tools workshop," Nov. 1999, http://www.cert.org/reports/dsit_workshop.pdf.

[26] Mixter, "Protecting against the unknown - a guide to improving network security to protect the Internet against future forms of security hazards," Jan. 2000, http://members.tripod.com/mixtersecurity/protecting.html.

[27] Jon Postel, "Internet protocol," Sept. 1981, RFC 791.

[28] K. Park and H. Lee, "A proactive approach to distributed DoS attack prevention using route-based distributed filtering," Tech. Rep. CSD-00-017, Department of Computer Sciences, Purdue University, December 2000.

[29] Wolfgang Theilmann and Kurt Rothermel, "Dynamic distance maps of the Internet," in Proc. of IEEE INFOCOM 2000, Mar. 2000.

[30] Josef Stoer and R. Bulirsch, Introduction to numerical anlaysis, New York: Springer-Verlag, 1993. 